\title{
Does Ras Activate Raf and PI3K Allosterically?
}

\author{
Ruth Nussinov ${ }^{1,2 *}$, Chung-Jung Tsai ${ }^{1}$ and Hyunbum Jang ${ }^{1}$ \\ ${ }^{1}$ Cancer and Inflammation Program, Leidos Biomedical Research, Inc., Frederick National Laboratory for Cancer Research, \\ National Cancer Institute at Frederick, Frederick, MD, United States, ${ }^{2}$ Department of Human Molecular Genetics and \\ Biochemistry, Sackler School of Medicine, Tel Aviv University, Tel Aviv, Israel
}

\section{OPEN ACCESS}

Edited by:

Georgia Konstantinidou, University of Bern, Switzerland

Reviewed by:

Roger Lee Williams, Medical Research Council,

United Kingdom Igor N. Berezovsky, Bioinformatics Institute (A*STAR), Singapore

*Correspondence: Ruth Nussinov NussinoR@mail.nih.gov

Specialty section This article was submitted to Molecular and Cellular Oncology,

a section of the journal Frontiers in Oncology

Received: 18 July 2019 Accepted: 28 October 2019 Published: 15 November 2019

Citation:

Nussinov R, Tsai C-J and Jang H (2019) Does Ras Activate Raf and PI3K Allosterically?

Front. Oncol. 9:1231 doi: 10.3389/fonc.2019.01231
The mechanism through which oncogenic Ras activates its effectors is vastly important to resolve. If allostery is at play, then targeting allosteric pathways could help in quelling activation of MAPK (Raf/MEK/ERK) and PI3K (PI3K/AKt/mTOR) cell proliferation pathways. On the face of it, allosteric activation is reasonable: Ras binding perturbs the conformational ensembles of its effectors. Here, however, we suggest that at least for Raf, PI3K, and NORE1A (RASSF5), that is unlikely. Raf's long disordered linker dampens effective allosteric activation. Instead, we suggest that the high-affinity Ras-Raf binding relieves Raf's autoinhibition, shifting Raf's ensemble from the inactive to the nanoclustermediated dimerized active state, as Ras also does for NORE1A. PI3K is recruited and allosterically activated by RTK (e.g., EGFR) at the membrane. Ras restrains PI3K's distribution and active site orientation. It stabilizes and facilitates $\mathrm{PIP}_{2}$ binding at the active site and increases the PI3K residence time at the membrane. Thus, RTKs allosterically activate PI3K $\alpha$; however, merging their action with Ras accomplishes full activation. Here we review their activation mechanisms in this light and draw attention to implications for their pharmacology.

Keywords: allosteric, allostery, B-Raf, KRas, K-Ras, NORE1A, BRAF

\section{INTRODUCTION}

Is allostery driving Ras activation of its effectors? The presumption that this is the case is easy to understand. Active Ras binds its effectors, and direct binding always perturbs the structures, initiating and promoting dynamic and at least some conformational changes (1-4). The relevant question is though-does Ras binding promote signals that propagate, through some allosteric pathways, and lead to a functional change? That is, do these signals prompt conformational and dynamic changes that affect the active site and are the dominant mechanism of effector activation? Even though not directly observed, the premise in the community has been that this is likely to be the case.

This premise has recently been revisited. Experimental and computational data indicated that at least for phosphatidylinositide-3-kinase $\alpha(\mathrm{PI} 3 \mathrm{~K} \alpha)$ this is not the case $(5,6)$. Indeed, PI3K $\alpha$ is known to be recruited and activated by epidermal growth factor receptor (EGFR), a receptor tyrosine kinase (RTK), at the membrane $(7,8)$. For Raf the premise still prevails. Here we overview $\mathrm{PI} 3 \mathrm{~K} \alpha$ and Raf activation, as well as activation of Ras association domain family 5 (RASSF5, a.k.a. NORE1A) tumor suppressor (Figure 1). We suggest that these Ras effectors are not activated via allosteric activation through Ras interaction. Further, even though to date there are no data relating to other Ras effectors, we suspect that this holds. In the case of Raf, a long disordered linker joins the kinase domain with the regulatory domain containing the Ras binding domain (RBD) and 


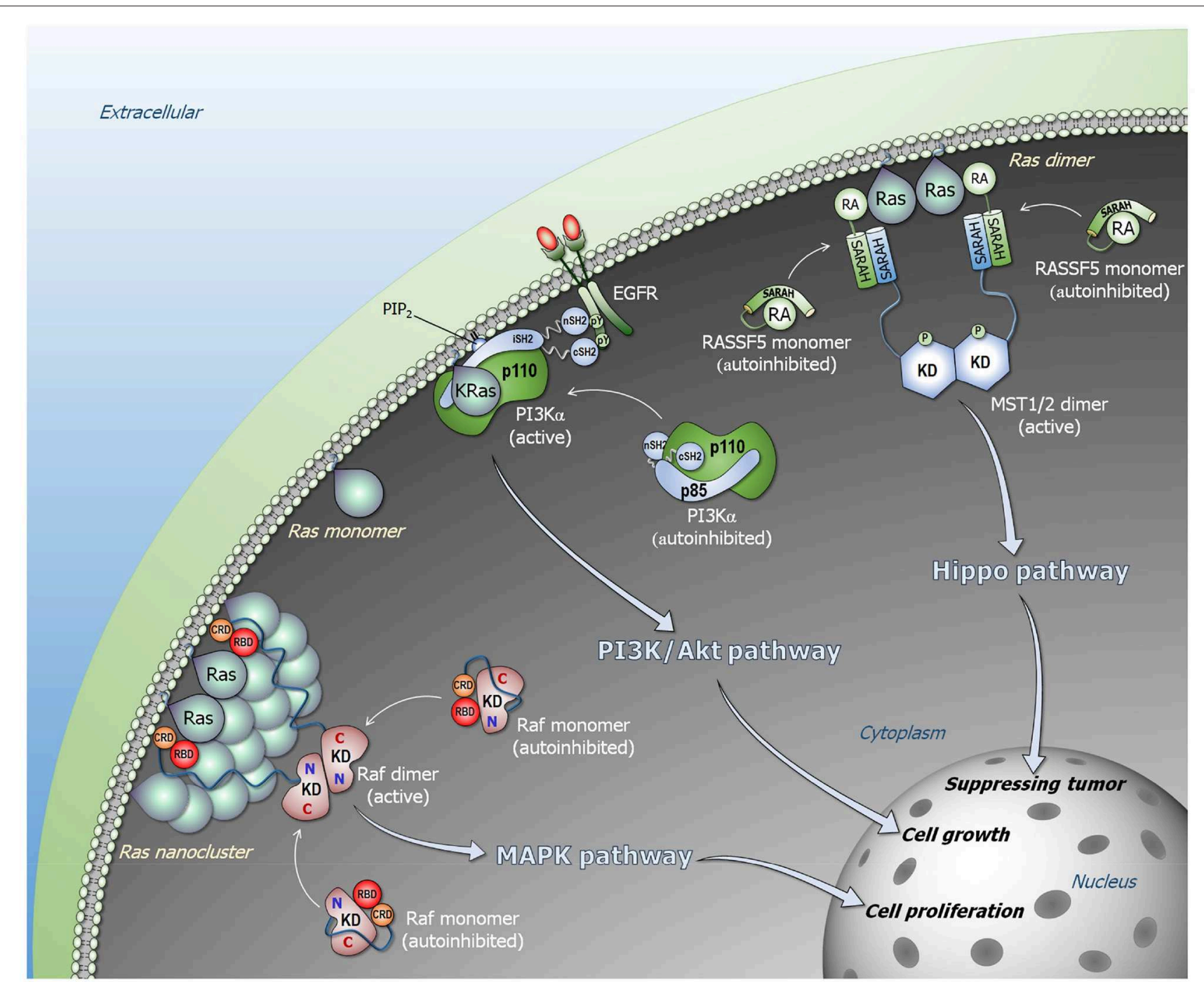

FIGURE 1 | Ras signaling pathways. Ras forms nanoclusters and promotes Raf dimerization in the Raf/MEK/ERK (MAPK) pathway (lower left). Monomeric Raf is autoinhibited in cytosol, and the high-affinity Ras-RBD interaction releases the autoinhibition, activating Raf through side-by-side dimerization. PI3K $\alpha$ is allosterically activated by EGFR (middle). The C-terminal phosphorylated tyrosine motif of EGFR liberates the SH2 domains of p85 $\alpha$ regulatory subunit from the p110 $\alpha$ catalytic subunit, releasing the autoinhibition of PI3K $\alpha$. Ras binding is not link to the allosteric activation of PI3K $\alpha$, but its binding contributes to further increase in the residence time of active PI3K $\alpha$ at the membrane. NORE1A (RASSF5) is an adaptor protein and autoinhibited by its RA domain interacting with its SARAH domain (upper right). In the presence of proximal Ras molecules, the Ras-RA interaction liberates NORE1A SARAH to recruit MST1/2 SARAH, promoting MST1/2 dimerization through their kinase domains that activates MST1/2 via cross phosphorylation. In the presence of Hippo signal, the active MST1/2 kinase promotes phosphorylation cascade signal, leading to YAP1 phosphorylation and degradation that result in tumor suppressing.

the cysteine-rich domain (CRD), which attaches Raf to the membrane (9-11). Protein disorder inherently implies no preferred interactions, no matter the sequence length. In the absence of specific interactions between the linker and RBD and the kinase domain, no allosteric propagation can take place. If no allosteric propagation, it is like there is no linkage between the two domains. The high-affinity Ras-RBD interaction $(12,13)-$ vs. the low affinity autoinhibition-argues in favor of activation via a shift in Raf's population toward the Ras-bound active state. In the case of PI3K $\alpha$, it is allosterically activated by the binding of the phosphorylated EGFR C-terminal motif to PI3K $\alpha$ 's Src homology 2 (SH2) domains $(7,14,15)$; not by Ras. These binding events promote a conformational change which relieves PI3K $\alpha$ autoinhibition and recruit $\mathrm{PI} 3 \mathrm{~K} \alpha$ to the membrane. Notably, EGFR activates PI3K $\alpha$ even in the absence of Ras (16), albeit to a lesser extent. Activation of NORE1A tumor suppressor resembles the activation of the Raf proteins $(17,18)$. Taken together, these lead us to suggest some guidelines as to when allostery may not be involved in activation in binding events. This is important, since the mechanisms of activation are considered in drug discovery (19-26). If allostery is at play, disrupting propagation pathways is often deliberated. 
Below, we first provide a brief background of allosteric activation. Next, we discuss activation of three Ras effectors, Raf, PI3K $\alpha$ and NORE1A, and why allostery is unlikely to be involved. Finally, we lay out guidelines relating to when allostery is unlikely.

\section{ALLOSTERIC ACTIVATION: DEFINITION AND BACKGROUND}

Classically, allosteric activation is defined as inducing a conformational change in the active site of the enzyme by binding at a location other than the active site. We suggested that if a conformational change is not observed, then it is likely due to limitations in the experimental approach used to detect a conformational change (27). Thus, with this definition, if Ras only has a role in recruiting the enzyme to the membrane, it would not be allostery since it does not elicit a conformation that alters the active site. Similarly, if Ras were to only restrict the orientation of the active site relative to the membrane to make productive catalysis more likely, by definition, this would also not be allostery because it would not involve a conformational change.

Allostery is linked to structural perturbation events (2737). The events can be covalent changes, such as mutations, allosteric post-translational modifications (PTMs) or covalent allosteric drugs (38-42), or non-covalent, such as binding of small molecules (drugs, membrane signaling lipids, cofactors, water molecules, ions) or macromolecules, such as proteins (4345). Allosteric events can take place near or away from the functional (active, protein-protein interaction, etc.) site; both can elicit efficient communication and productive allosteric events $(29,46,47)$. Whether covalent or non-covalent, the perturbation breaks and forms new atomic interactions. In turn, the local changes promote additional adjustments in the interactions in their environments. These remodeling perturbations propagate along multiple pathways, with favored paths extending to the functional site, shifting the ensemble, thereby accomplishing distinct conformational and dynamic changes that switch the protein from the inactive to the active state (vice versa for repressors) (Figure 2). Thus, conformational dynamics is implicitly at play since allosteric events take place by a shift of the ensemble from energetically less favored states to more favored ones. Notably, the active conformation already exists in the ensemble; however, the shifts in the ensemble that allostery promotes increase its population. This conformation is primed to bind the substrate.

Allostery involves propagation which argues that the location of the allosteric event with respect to the active site is an important factor in determining its efficiency. Even though compact structures can act as efficient vehicles in allosteric transmission, dynamic segments, such as loops, linkers and hinges, respond and can efficiently mediate function $(48,49)$. Ras effectors are multidomain proteins, and to date no statistics have been published of the distributions of cancer driver mutations in multidomain proteins with respect to the functional (active) site. We expect that driver mutations tend to occur in the domain whose function is targeted. Mutations occurring in the catalytic domain make the active site conformation substrate-favored; those in a regulatory domain that acts in autoinhibition through its interaction with the catalytic domain, would relieve the autoinhibition. We are unaware of driver mutations occurring in non-catalytic domains whose actions propagate via disordered linkers to alter the active site conformations, as would be the case if Ras binding to the Raf's RBD were to allosterically activate it. To our knowledge, to date no driver mutations have been identified in Raf's RBD to substitute for its interaction with Ras.

To explain how Ras activates Raf, we consider two fundamental physical tenets. First, every biomacromolecule exists in an ensemble of conformations. For rigid molecules the ensemble is more restricted; for flexible (especially disordered) it is broad. Second, the most stable state is the most populated state. The ensemble of Raf monomers can be classified into three states: an active Ras-bound "open" state; a free "open" conformational state, and an autoinhibited "closed" state, where the kinase domain is blocked by another segment of Raf which prohibits it from dimerization (Figure 1). In the absence of Ras, Raf largely populates the microensemble of the autoinhibited state; however, a certain fraction of the population will be in the free state. The autoinhibited state is unlikely to be stable, since if it were, it should be possible to experimentally determine it (by crystallization, NMR). This is not the case for the very stable Ras-RBD complex. In the presence of Ras, Raf is most highly populated in the Ras-bound state due to a shift of the free state fraction. The equilibrium between the autoinhibited state and the free state will then be restored by a certain shift of the autoinhibited state to the free state. Kinase domain dimerization can take place even in the absence of Ras; however, GTP-bound active Ras raises the otherwise low population of the active species, with the exposed kinase domain prepped for dimerization. Ras' action in NORE1A's activation resembles its action in Raf's activation (Figure 1).

Allostery is unlikely to be at play in Ras' contribution to $\mathrm{PI} 3 \mathrm{~K} \alpha$ activation either. RTK binds PI3K $\alpha$ (Figure 1). Binding promotes relief of PI3K $\alpha$ 's autoinhibition and exposure of the active site to the lipid substrate at the membrane through conformational change (6). However, no conformational change in $\mathrm{PI} 3 \mathrm{~K} \alpha$ is stimulated by Ras. Consequently, it is reasonable to conclude that the mechanism of Ras' activation of PI3K $\alpha$ is not allosteric. Thus, even though the mechanisms of Ras activation of its effectors differ, in none of those explored here allostery is incurred by Ras action. Below we provide the mechanistic details.

\section{ACTIVATION OF RAS EFFECTORS RAF, PI3K AND NORE1A}

\section{If Not Allostery, What Is Ras Role in PI3K $\alpha$ Activation?}

$\mathrm{PI} 3 \mathrm{~K} \alpha$ is a lipid kinase that phosphorylates phosphatidylinositol 4,5-bisphosphate $\left(\mathrm{PIP}_{2}\right)$ to phosphatidylinositol 3,4,5trisphosphate $\left(\mathrm{PIP}_{3}\right)$. Binding of Akt protein kinase to $\mathrm{PIP}_{3}$ at the membrane is a key step in the AkT/mTOR signaling pathway leading to cell growth and proliferation. Inactive $\mathrm{PI} 3 \mathrm{~K} \alpha$ is a 


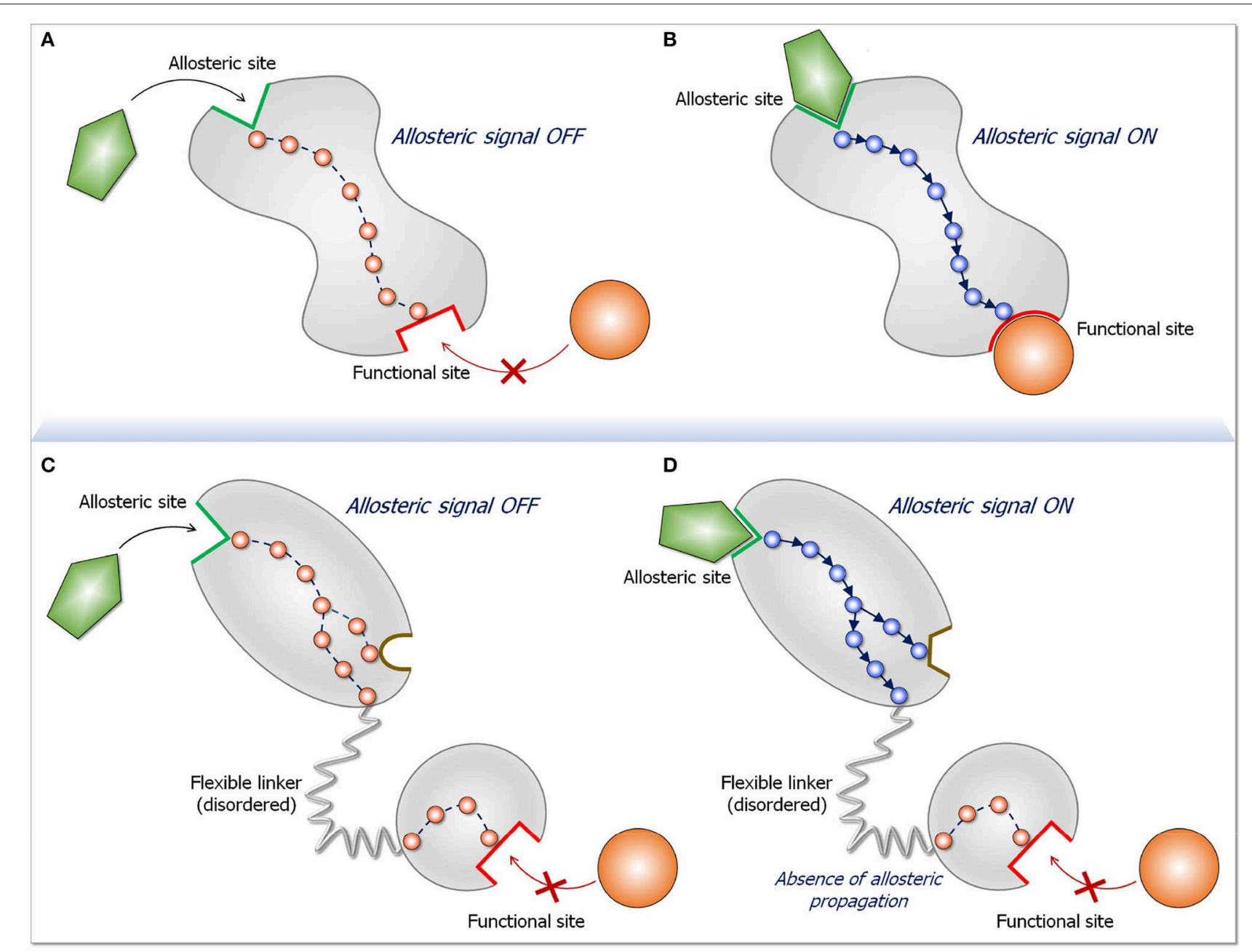

FIGURE 2 | Schematic diagram for an allosteric propagation pathway and its absence in long disordered linkers. The top two panels display a two-state dynamic allosteric switch. Both states pre-exist in the population. In the absence of the ligand (A) the protein populates a conformation in the ligand-free state. Upon ligand binding at the allosteric site (B), a functional switch that is in favor of a ligand-bound state initiates at the binding site and propagates down to the functional site. The two bottom panels (C,D) depict what happens when two domains are joined by a long, disordered linker. The two-state switch takes place only in the domain to which the allosteric ligand binds, but do not propagate down the linker. The reason for the absence of allosteric propagation through the long linker is that the disordered state is distributed in multiple conformations. Since in the disordered state there are no specific stabilized interactions, there is no preferred propagation pathway. Preferred propagation pathways are required for population shift. In practice, identification of an allosteric propagation pathway in the structure can be achieved through superposition of the two (active and inactive) structures and locating changes in interactions of residues along pathways extending from the allosteric site to the functional site.

stable heterodimer. It consists of the $\mathrm{p} 85 \alpha$ regulatory subunit and $\mathrm{p} 110 \alpha$ catalytic subunit $(6,50)$ whose active site is blocked by $\mathrm{p} 85 \alpha$ (15). Conformational changes, elicited primarily by the $\mathrm{nSH} 2$ domain of $\mathrm{p} 85 \alpha$, are a key step in $\mathrm{PI} 3 \mathrm{~K} \alpha$ activation $(51,52)$. These are the outcome of allosteric perturbation by EGFR (or another RTK). The phosphorylated tyrosine motif (pYxxM) in the C-terminal of RTK, interacts with high affinity with the nSH2 domain $(7,14)$. This interaction breaks the nSH2-p110 $\alpha$ helical interface eliciting a conformational change that releases the $\mathrm{nSH} 2$ from $\mathrm{p} 110 \alpha$, as well as the $\mathrm{p} 85 \alpha$ iSH 2 domain from the $\mathrm{p} 110 \alpha$

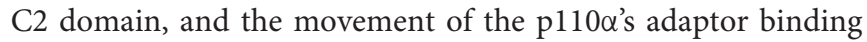
domain (ABD). iSH2 forms strong hydrophobic interactions and salt bridges with p110 's ABD, C2 and the kinase domains. Its rotation breaks its interaction with $\mathrm{p} 110 \alpha$ 's ABD consistent with hydrogen deuterium exchange mass spectrometry (HDX-MS) data (53). These conformational changes expose the $\mathrm{PI} 3 \mathrm{~K} \alpha$ membrane binding surface $(5,53-55)$. The mechanism of $\mathrm{PI} 3 \mathrm{~K} \alpha$ activation that we determined underscores the action of the RTK motif via its interaction with the $\mathrm{nSH} 2$ and the associated large conformational change. The release of $\mathrm{nSH} 2$ permits the $\mathrm{C}$-lobe of the kinase domain to get away from the $\mathrm{C} 2$ domain, priming PI3K $\alpha$ for phosphorylation of the PIP 2 lipid substrate to $\mathrm{PIP}_{3}$ $(15,56)$. In oncogenic Ras, in the absence of RTK, calmodulin (CaM)'s phosphorylated tyrosine can similarly target the $\mathrm{nSH} 2$ (and cSH2 domains), recruiting and activating PI3K $\alpha$ (57-59). Alternatively, EGFR overexpression can take place. 
What is then Ras' role in PI3K $\alpha$ activation? The RTK motif already accomplishes recruitment to the membrane with the coupled conformational change that relieves the autoinhibition and switches it from the inactive to the active state. The conformational change created by Ras binding is insignificant, and unlikely to play a role in activation. However, the PI3K $\alpha$ population which is favorably positioned and oriented, primed for substrate binding and catalysis, is limited. We conclude that Ras binding serves to further increase the PI3K residence time at the membrane, stabilizing and facilitating $\mathrm{PIP}_{2}$ binding at the active site. Thus, RTKs allosterically activate PI3K $\alpha$; however, merging their action with Ras accomplishes full activation (5).

\section{If Not Allostery, How Does Ras Activate Raf?}

Raf is a multidomain protein. It has a variable length $\mathrm{N}$ terminal tail that was proposed to mediate calcium-dependent B-Raf homo- and hetero-dimerization (60), interact with the Cterminal (61), and be responsible for A-Raf low basal activity. It also includes the RBD and CRD domain that latches Raf to the membrane, a variable-length linker containing the Ser/Thrrich segment $(10,11)$, and the kinase domain. In the inactive state, monomeric Raf is autoinhibited. It's likely autoinhibited organization has recently been reviewed (9) along with the supporting experimental data and theoretical considerations (11, 61-87).

The high affinity (nanomolar range) active Ras-Raf's RBD binding recruits Raf to the plasma membrane $(61,88)$. CRD's anchorage to the membrane (89-91) is stabilized by its 'membrane insertion' loop residues $(89,92)$ in an organization that is similar to the one it adopts when alone, not in the RasRBD context (89). The Raf-1 linker connecting RBD and CRD consists of only 6 residues that further constrain and stabilize the Ras-RDB-CRD organization at the membrane. No interactions are observed between KRas4B, including the farnesyl, and CRD. This is not the case for the HRas farnesyl group. However, different than KRas, HRas has also two palmitoyls, and the two membrane-anchored palmitoyls lend stability to the system (93). Additional interaction details of the different Ras-Raf systems have also been uncovered $(59,89,94-96)$. In a favored orientation, KRas4B attaches to the membrane through its farnesylated hypervariable region (HVR) in a way such that the effector binding site faces away from the membrane and is largely exposed. This permits the RBD to interact at the effector binding site while the CRD is anchored at the membrane through its loop. The nanomolar affinity of the Ras-RBD interaction has been measured in solution. However, under physiological conditions at the membrane, fluctuations that take place and molecular dynamics (MD) simulations indicate that these can be significant. The tethered Ras-RBD-CRD organization reduces the Ras-RBD fluctuations, thus increases the residence times of the productive organization. The enhanced affinity promotes a population shift of the Raf ensemble toward this Ras-bound state, relieving the autoinhibition.

High affinity is not the sole factor controlling the relief of Raf's autoinhibition and population shift toward the open state. Whereas, the disordered linker $(\sim 180$ residues in B-Raf; $\sim 170$ residues in Raf-1) between CRD and the kinase domain deters allosteric transmission, it also encodes residues whose phosphorylation enhances or abrogates the autoinhibition. Ser446 phosphorylation of B-Raf weakens the autoinhibition; phosphorylated Ser259 of Raf-1 is recognized by 14-3-3 proteins $(86,87,97,98)$, promoting the autoinhibition. Dephosphorylation by protein phosphatase 2A (PP2A) and protein phosphatase 1 (PP1) releases it, shifting the equilibrium toward open state (11, 80, 99-101). 14-3-3 also binds phosphorylated Ser621 of Raf-1 (86, 97, 98). The interaction of the N-terminal with the kinase domain is likely to be weak (9). Simultaneous binding at both sites can promote the autoinhibited state by stabilizing the interaction of the $\mathrm{N}$ terminal segment and the kinase domain (11, 73, 87, 102-104). However, these distinct sites that assist in regulating the switch controlling the On/Off open/closed states, may not need such long linkers.

Taken together, this raises the question of why long linkers? We believe that the long linkers permit distancing the kinase domains from Ras-RBD-CRD at the membrane. The membrane is crowded. The linker efficiently connects the protein assemblies at the cytoplasm with signals communicated through receptor proteins, such as RTKs. In the cytoplasm, dimers of Raf kinase domains gather in large complexes, including mitogen-activated protein kinase (MEK) and extracellular signal-regulated kinase (ERK) dimers. Large scaffolding and adaptor proteins are also involved, e.g., kinase suppressor of Ras (KSR) (105, 106), IQ motif-containing GTPase activating protein (IQGAP) (107), heat shock protein (HSP90) (108), and galectin (109). All are large multidomain proteins that interact with additional proteins, such as IQGAP1 with Arp2/3 which stimulates branching of actin assemblies (110). The long linker provides an effective and pragmatic solution, enabling formation of clusters in the cytoplasm thus signaling efficiency. The large clusters are further favored by the water layer at the membrane surface which "pushes" or drives the proteins away from the membrane surface unless there are lipid-favoring residues at the protein surface, as in the case of CRD. The long linkers also vacate the requirement for Ras dimerization for Raf's activation. They allow Ras nanoclusters-mediated Raf's dimerization and activation (Figure 1).

Thus, rather than allostery, current data argues for a shift of the ensemble through release of the autoinhibited, closed state. In the absence of active Ras molecules, Raf mostly populates a closed autoinhibited state, with access to the kinase domain hindered by other segments. In the presence of Ras, the high affinity Ras-RBD interaction at the membrane shifts the ensemble. This mechanism is also supported by the dual 14-33 interaction, phosphorylation (dephosphorylation) experiments and mutational data [e.g., alanine and acidic substitutions at phosphorylation sites in the activation loop (73-75)]. It can explain why Raf evolved tight interaction with Ras and why Ras nanoclusters can function effectively in Raf's activation (111). It can also clarify how the large Raf assemblies with MAPK kinases and scaffolding proteins can form, act efficiently (112), and allow signaling dynamics (113) despite the crowded membrane surface. 


\section{If Not Allostery, How Does Ras Activate NORE1A?}

Different from Raf and PI3K $\alpha$, NORE1A (RASSF5) Ras effector is not a kinase, but essentially an adaptor protein, mediating the interactions of Ras and mammalian sterile 20like kinase $1 / 2$ (MST1/2). Ras-bound NORE1A activates the MST1/2 kinase (17, 114-118), which via the Hippo pathway phosphorylation cascade, leads to Yes-associated protein 1 (YAP1) phosphorylation and degradation. Overexpression of YAP1 induces cell proliferation (119). In the absence of active Ras, it is in a closed conformation, with its Ras association (RA) domain interacting weakly with the Sav-RASSF-Hippo (SARAH) domain. The linker between the two domains is short (5 residues) and contains a flexible hinge. In the presence of active Ras, the equilibrium shifts in favor of the tight Ras-RA interaction. The dissociated SARAH domain heterodimerizes with the MST1/2 SARAH domain. The tightly bound SARAH domain heterodimer releases the MST from its autoinhibited state, where the kinase domain interacts weakly with the MST SARAH domain. This shift in the MST ensemble from the inactive closed state to the open state permits kinase domain homodimerization and activation via trans-autophosphorylation. The affinity of the MST1/2 SARAH homodimer is lower than that of the heteroSARAH dimer $(120,121)$, putting it under Ras control. NORE1A bridges Ras and MST (17), with Ras interaction acting to bring MST1/2 kinase domains into spatial proximity $(18,122)$, just like it activates Raf. Thus, rather than allostery activating NORE1A to promote its activation of MST kinase, the high (micromolar) affinity of the SARAH heterodimer drives the equilibrium toward NORE1A open active state, driving MST1/2 kinase activation via population shift.

\section{CONCLUDING REMARKS}

Conformational ensembles and their shifts underlie biological processes (1-4, 30, 32, 123-131). Population shifts between two states due to differences in the stabilities follow the thermodynamic rule that systems are always driven to their free energy minima. In the case of the two Ras effectors described here, Raf and NORE1A, the higher stability of the

\section{REFERENCES}

1. Tsai CJ, Nussinov R. A unified view of "how allostery works". PLoS Comput Biol. (2014) 10:e1003394. doi: 10.1371/journal.pcbi.1003394

2. Liu J, Nussinov R. Allostery: an overview of its history, concepts, methods, and applications. PLoS Comput Biol. (2016) 12:e1004966. doi: 10.1371/journal.pcbi.1004966

3. Nussinov R. Introduction to protein ensembles and allostery. Chem Rev. (2016) 116:6263-6. doi: 10.1021/acs.chemrev.6b00283

4. Boehr DD, Nussinov R, Wright PE. The role of dynamic conformational ensembles in biomolecular recognition. Nat Chem Biol. (2009) 5:78996. doi: 10.1038/nchembio. 232

5. Buckles TC, Ziemba BP, Masson GR, Williams RL, Falke JJ. Single-molecule study reveals how receptor and ras synergistically activate PI3Kalpha and PIP3 signaling. Biophys J. (2017) 113:2396-405. doi: 10.1016/j.bpj.2017.09.018 interaction with Ras vs. that of the autoinhibited state drives the changes in the equilibrium. In the third, PI3K, Ras increases the population time at the membrane, facilitating $\mathrm{PIP}_{2}$ insertion. Understanding how Ras effectors are regulated is of paramount importance since it can help in pharmacological discovery. Ras has additional effectors, including Tiam1, RalGDS, AF6, RIN, and more. Scenarios involving high affinity to Ras and long disordered interdomain linkers are likely to discourage allosteric transmission. A tell-tale is the presence (or absence) of observable conformational changes $(27,132)$. If binding promotes a conformational change, allostery is likely at play (Figure 2). This is the case for RTK's phosphorylated motif promoting conformational change in the interactions of the $\mathrm{nSH} 2$ domain of the $\mathrm{p} 85 \alpha$, which expose p110 $\alpha$ active site. On the other hand, in our MD simulations of PI3K $\alpha$ RBD complexed with KRas4B, we observed only insignificant conformational changes in RBD making an allosteric mechanism unlikely, in line with experimental data discussed here.

Finally, Ras does not have an allosteric role for the three effectors discussed above. However, this is not necessarily always the case for Ras or Ras-like GTPases. One example is the Ras family GTPase RHEB that appears to have a primary role as an allosteric activator of the mTORC1 complex (133).

\section{AUTHOR CONTRIBUTIONS}

All authors listed have made a substantial, direct and intellectual contribution to the work, and approved it for publication.

\section{FUNDING}

This project has been funded in whole or in part with federal funds from the National Cancer Institute, National Institutes of Health, under contract HHSN261200800001E. The content of this publication does not necessarily reflect the views or policies of the Department of Health and Human Services, nor does mention of trade names, commercial products or organizations imply endorsement by the US Government. This research was supported [in part] by the Intramural Research Program of NIH, National Cancer Institute, Center for Cancer Research.
6. Zhang $\mathrm{M}$, Jang $\mathrm{H}$, Nussinov $\mathrm{R}$. The structural basis for Ras activation of PI3Kalpha lipid kinase. Phys Chem Chem Phys. (2019) 21:12021-8. doi: 10.1039/C9CP $00101 \mathrm{H}$

7. Nolte RT, Eck MJ, Schlessinger J, Shoelson SE, Harrison SC. Crystal structure of the PI 3-kinase p85 amino-terminal SH2 domain and its phosphopeptide complexes. Nat Struct Biol. (1996) 3:364-74. doi: 10.1038/nsb0496-364

8. Gabelli SB, Echeverria I, Alexander M, Duong-Ly KC, ChavesMoreira D, Brower ET, et al. Activation of PI3Kalpha by physiological effectors and by oncogenic mutations: structural and dynamic effects. Biophys Rev. (2014) 6:89-95. doi: 10.1007/s12551-01 3-0131-1

9. Nussinov R, Zhang M, Tsai CJ, Liao TJ, Fushman D, Jang H. Autoinhibition in Ras effectors Raf, PI3Kalpha, and RASSF5: a comprehensive review underscoring the challenges in pharmacological intervention. Biophys Rev. (2018) 10:1263-82. doi: 10.1007/s12551-018-0461-0 
10. Terrell EM, Morrison DK. Ras-mediated activation of the raf family kinases. Cold Spring Harb Perspect Med. (2019) 9:a033746. doi: 10.1101/cshperspect.a033746

11. Lavoie H, Therrien M. Regulation of RAF protein kinases in ERK signalling. Nat Rev Mol Cell Biol. (2015) 16:281-98. doi: 10.1038/nrm3979

12. Chuang E, Barnard D, Hettich L, Zhang XF, Avruch J, Marshall MS. Critical binding and regulatory interactions between Ras and Raf occur through a small, stable N-terminal domain of Raf and specific Ras effector residues. Mol Cell Biol. (1994) 14:5318-25. doi: 10.1128/MCB.14.8.5318

13. Herrmann C, Martin GA, Wittinghofer A. Quantitative analysis of the complex between p21ras and the Ras-binding domain of the human Raf-1 protein kinase. J Biol Chem. (1995) 270:2901-5. doi: 10.1074/jbc.270.7.2901

14. Pauptit RA, Dennis CA, Derbyshire DJ, Breeze AL, Weston SA, Rowsell S, Murshudov GN. NMR trial models: experiences with the colicin immunity protein $\operatorname{Im} 7$ and the p85alpha C-terminal SH2peptide complex. Acta Crystallogr D Biol Crystallogr. (2001) 57:1397404. doi: 10.1107/S0907444901012434

15. Zhang M, Jang H, Nussinov R. The mechanism of PI3Kalpha activation at the atomic level. Chem Sci. (2019) 10:3671-80. doi: 10.1039/C8SC04498H

16. Karasarides M, Anand-Apte B, Wolfman A. A direct interaction between oncogenic Ha-Ras and phosphatidylinositol 3-kinase is not required for Ha-Ras-dependent transformation of epithelial cells. J Biol Chem. (2001) 276:39755-64. doi: 10.1074/jbc.M102401200

17. Liao TJ, Tsai CJ, Jang H, Fushman D, Nussinov R. RASSF5: an MST activator and tumor suppressor in vivo but opposite in vitro. Curr Opin Struct Biol. (2016) 41:217-24. doi: 10.1016/j.sbi.2016.09.001

18. Liao TJ, Jang H, Tsai CJ, Fushman D, Nussinov R. The dynamic mechanism of RASSF5 and MST kinase activation by Ras. Phys Chem Chem Phys. (2017) 19:6470-80. doi: 10.1039/C6CP08596B

19. Stephens L, Williams R, Hawkins P. Phosphoinositide 3kinases as drug targets in cancer. Curr Opin Pharmacol. (2005) 5:357-65. doi: 10.1016/j.coph.2005.03.002

20. Jambrina PG, Bohuszewicz O, Buchete NV, Kolch W, Rosta E. Molecular mechanisms of asymmetric RAF dimer activation. Biochem Soc Trans. (2014) 42:784-90. doi: 10.1042/BST20140025

21. Joseph EW, Pratilas CA, Poulikakos PI, Tadi M, Wang W, Taylor BS, et al. The RAF inhibitor PLX4032 inhibits ERK signaling and tumor cell proliferation in a V600E BRAF-selective manner. Proc Natl Acad Sci USA. (2010) 107:14903-8. doi: 10.1073/pnas.1008990107

22. Poulikakos PI, Zhang C, Bollag G, Shokat KM, Rosen N. RAF inhibitors transactivate RAF dimers and ERK signalling in cells with wild-type BRAF. Nature. (2010) 464:427-30. doi: 10.1038/nature08902

23. Vadas O, Burke JE, Zhang X, Berndt A, Williams RL. Structural basis for activation and inhibition of class I phosphoinositide 3-kinases. Sci Signal. (2011) 4:re2. doi: 10.1126/scisignal.2002165

24. Ruan Z, Kannan N. Altered conformational landscape and dimerization dependency underpins the activation of EGFR by alphaCbeta4 loop insertion mutations. Proc Natl Acad Sci USA. (2018) 115:E8162-71. doi: 10.1073/pnas.1803152115

25. Hubbard PA, Moody CL, Murali R. Allosteric modulation of Ras and the PI3K/AKT/mTOR pathway: emerging therapeutic opportunities. Front Physiol. (2014) 5:478. doi: 10.3389/fphys.2014.00478

26. Tsai CJ, Nussinov R. Allosteric activation of RAF in the MAPK signaling pathway. Curr Opin Struct Biol. (2018) 53:100-6. doi: 10.1016/j.sbi.2018.07.007

27. Nussinov R, Tsai CJ. Allostery without a conformational change? Revisiting the paradigm. Curr Opin Struct Biol. (2015) 30:17-24. doi: 10.1016/j.sbi.2014.11.005

28. Guo J, Zhou HX. Protein allostery and conformational dynamics. Chem Rev. (2016) 116:6503-15. doi: 10.1021/acs.chemrev.5b00590

29. Smith IN, Thacker S, Seyfi M, Cheng F, Eng C. Conformational dynamics and allosteric regulation landscapes of germline PTEN mutations associated with autism compared to those associated with cancer. Am J Hum Genet. (2019) 104:861-78. doi: 10.1016/j.ajhg.2019.03.009

30. Nussinov R, Tsai CJ, Ma B. The underappreciated role of allostery in the cellular network. Annu Rev Biophys. (2013) 42:169-89. doi: 10.1146/annurev-biophys-083012-130257
31. Nussinov R, Tsai CJ. Allostery in disease and in drug discovery. Cell. (2013) 153:293-305. doi: 10.1016/j.cell.2013.03.034

32. Del Sol A, Tsai CJ, Ma B, Nussinov R. The origin of allosteric functional modulation: multiple pre-existing pathways. Structure. (2009) 17:104250. doi: 10.1016/j.str.2009.06.008

33. Gardino AK, Villali J, Kivenson A, Lei M, Liu CF, Steindel P, et al. Transient non-native hydrogen bonds promote activation of a signaling protein. Cell. (2009) 139:1109-18. doi: 10.1016/j.cell.2009.11.022

34. Astl L, Verkhivker GM. Atomistic Modeling of the ABL kinase regulation by allosteric modulators using structural perturbation analysis and communitybased network reconstruction of allosteric communications. J Chem Theory Comput. (2019) 15:3362-80. doi: 10.1021/acs.jctc.9b00119

35. Pfleger C, Minges A, Boehm M, Mcclendon CL, Torella R, Gohlke H. Ensemble- and rigidity theory-based perturbation approach to analyze dynamic allostery. J Chem Theory Comput. (2017) 13:6343-57. doi: 10.1021/acs.jctc.7b00529

36. Ettayapuram Ramaprasad AS, Uddin S, Casas-Finet J, Jacobs DJ. Decomposing dynamical couplings in mutated scFv antibody fragments into stabilizing and destabilizing effects. J Am Chem Soc. (2017) 139:1750817. doi: 10.1021/jacs.7b09268

37. Saleh N, Saladino G, Gervasio FL, Clark T. Investigating allosteric effects on the functional dynamics of beta2-adrenergic ternary complexes with enhanced-sampling simulations. Chem Sci. (2017) 8:4019-26. doi: 10.1039/C6SC04647A

38. Onel M, Sumbul F, Liu J, Nussinov R, Haliloglu T. Cullin neddylation may allosterically tune polyubiquitin chain length and topology. Biochem J. (2017) 474:781-95. doi: 10.1042/BCJ20160748

39. Zhan C, Qi R, Wei G, Guven-Maiorov E, Nussinov R, Ma B. Conformational dynamics of cancer-associated MyD88-TIR domain mutant L252P (L265P) allosterically tilts the landscape toward homo-dimerization. Protein Eng Des Sel. (2016) 29:347-54. doi: 10.1093/protein/gzw033

40. Nussinov R, Tsai CJ. The design of covalent allosteric drugs. Annu Rev Pharmacol Toxicol. (2015) 55:24967. doi: 10.1146/annurev-pharmtox-010814-124401

41. Lu S, Zhang J. Designed covalent allosteric modulators: an emerging paradigm in drug discovery. Drug Discov Today. (2017) 22:447-53. doi: 10.1016/j.drudis.2016.11.013

42. Bradshaw JM, Mcfarland JM, Paavilainen VO, Bisconte A, Tam D, Phan VT, et al. Prolonged and tunable residence time using reversible covalent kinase inhibitors. Nat Chem Biol. (2015) 11:525-31. doi: 10.1038/nchembio.1817

43. Tsai CJ, Nussinov R. Emerging allosteric mechanism of EGFR activation in physiological and pathological contexts. Biophys J. (2019) 117:513. doi: $10.1016 /$ j.bpj.2019.05.021

44. Zhao J, Nussinov R, Ma B. Antigen binding allosterically promotes Fc receptor recognition. MAbs. (2019) 11:5874. doi: 10.1080/19420862.2018.1522178

45. Liao TJ, Jang H, Fushman D, Nussinov R. Allosteric KRas4B can modulate SOS1 Fast and slow ras activation cycles. Biophys J. (2018) 115:62941. doi: 10.1016/j.bpj.2018.07.016

46. Verkhivker GM. Biophysical simulations and structure-based modeling of residue interaction networks in the tumor suppressor proteins reveal functional role of cancer mutation hotspots in molecular communication. Biochim Biophys Acta Gen Subj. (2019) 1863:210-25. doi: 10.1016/j.bbagen.2018.10.009

47. Daily MD, Upadhyaya TJ, Gray JJ. Contact rearrangements form coupled networks from local motions in allosteric proteins. Proteins. (2008) 71:45566. doi: $10.1002 /$ prot. 21800

48. Papaleo E, Saladino G, Lambrughi M, Lindorff-Larsen K, Gervasio FL, Nussinov R. The role of protein loops and linkers in conformational dynamics and allostery. Chem Rev. (2016) 116:6391-423. doi: 10.1021/acs.chemrev.5b00623

49. Ma B, Tsai CJ, Haliloglu T, Nussinov R. Dynamic allostery: linkers are not merely flexible. Structure. (2011) 19:907-17. doi: 10.1016/j.str.2011.06.002

50. Thorpe LM, Spangle JM, Ohlson CE, Cheng H, Roberts TM, Cantley LC, Zhao JJ. PI3K-p110alpha mediates the oncogenic activity induced by loss of the novel tumor suppressor PI3K-p85alpha. Proc Natl Acad Sci USA. (2017) 114:7095-100. doi: 10.1073/pnas.1704706114 
51. Castellano E, Downward J. RAS Interaction with PI3K: more than just another effector pathway. Genes Cancer. (2011) 2:261-74. doi: 10.1177/1947601911408079

52. Murillo MM, Zelenay S, Nye E, Castellano E, Lassailly F, Stamp G, Downward J. RAS interaction with PI3K p110alpha is required for tumor-induced angiogenesis. J Clin Invest. (2014) 124:3601-11. doi: 10.1172/JCI74134

53. Burke JE, Perisic O, Masson GR, Vadas O, Williams RL. Oncogenic mutations mimic and enhance dynamic events in the natural activation of phosphoinositide 3-kinase p110alpha (PIK3CA). Proc Natl Acad Sci USA. (2012) 109:15259-64. doi: 10.1073/pnas.1205508109

54. Tsutsumi K, Fujioka Y, Tsuda M, Kawaguchi H, Ohba Y. Visualization of Ras-PI3K interaction in the endosome using BiFC. Cell Signal. (2009) 21:1672-9. doi: 10.1016/j.cellsig.2009.07.004

55. Wang J, Yuan Y, Zhou Y, Guo L, Zhang L, Kuai X, et al. Protein interaction data set highlighted with human Ras-MAPK/PI3K signaling pathways. $J$ Proteome Res. (2008) 7:3879-89. doi: 10.1021/pr8001645

56. Jang H, Banerjee A, Chavan T, Gaponenko V, Nussinov R. Flexiblebody motions of calmodulin and the farnesylated hypervariable region yield a high-affinity interaction enabling K-Ras4B membrane extraction. J Biol Chem. (2017) 292:12544-59. doi: 10.1074/jbc.M117. 785063

57. Nussinov R, Tsai CJ, Jang H. Oncogenic KRas mobility in the membrane and signaling response. Semin Cancer Biol. (2019) 54:109-13. doi: 10.1016/j.semcancer.2018.02.009

58. Chavan TS, Jang H, Khavrutskii L, Abraham SJ, Banerjee A, Freed BC, et al. High-Affinity Interaction of the K-Ras4B Hypervariable Region with the Ras Active Site. Biophys J. (2015) 109:2602-13. doi: 10.1016/j.bpj.2015.09.034

59. Jang H, Banerjee A, Chavan TS, Lu S, Zhang J, Gaponenko V, et al. The higher level of complexity of K-Ras4B activation at the membrane. FASEB J. (2016) 30:1643-55. doi: 10.1096/fj.15-2 79091

60. Terai K, Matsuda M. The amino-terminal B-Raf-specific region mediates calcium-dependent homo- and hetero-dimerization of Raf. EMBO J. (2006) 25:3556-64. doi: 10.1038/sj.emboj.7601241

61. Chong H, Guan KL. Regulation of Raf through phosphorylation and $\mathrm{N}$ terminus-C terminus interaction. J Biol Chem. (2003) 278:3626976. doi: 10.1074/jbc.M212803200

62. Bruder JT, Heidecker G, Rapp UR. Serum-, TPA-, and Ras-induced expression from Ap-1/Ets-driven promoters requires Raf-1 kinase. Genes Dev. (1992) 6:545-56. doi: 10.1101/gad.6.4.545

63. Fukui M, Yamamoto $\mathrm{T}$, Kawai $\mathrm{S}$, Mitsunobu $\mathrm{F}$, Toyoshima $\mathrm{K}$. Molecular cloning and characterization of an activated human craf-1 gene. Mol Cell Biol. (1987) 7:1776-81. doi: 10.1128/MCB.7. 5.1776

64. Heidecker G, Huleihel M, Cleveland JL, Kolch W, Beck TW, Lloyd P, et al. Mutational activation of c-raf- 1 and definition of the minimal transforming sequence. Mol Cell Biol. (1990) 10:2503-12. doi: 10.1128/MCB.10. 6.2503

65. Ishikawa F, Sakai R, Ochiai M, Takaku F, Sugimura T, Nagao M. Identification of a transforming activity suppressing sequence in the c-raf oncogene. Oncogene. (1988) 3:653-8.

66. Ishikawa F, Takaku F, Hayashi K, Nagao M, Sugimura T. Activation of rat c-raf during transfection of hepatocellular carcinoma DNA. Proc Natl Acad Sci USA. (1986) 83:3209-12. doi: 10.1073/pnas.83. 10.3209

67. Molders H, Defesche J, Muller D, Bonner TI, Rapp UR, Muller R. Integration of transfected LTR sequences into the c-raf proto-oncogene: activation by promoter insertion. EMBO J. (1985) 4:693-8. doi: 10.1002/j.1460-2075.1985.tb 03685.x

68. Schultz AM, Copeland T, Oroszlan S, Rapp UR. Identification and characterization of c-raf phosphoproteins in transformed murine cells. Oncogene. (1988) 2:187-93.

69. Stanton VP Jr, Cooper GM. Activation of human raf transforming genes by deletion of normal amino-terminal coding sequences. Mol Cell Biol. (1987) 7:1171-9. doi: 10.1128/MCB.7.3.1171
70. Stanton VP Jr, Nichols DW, Laudano AP, Cooper GM. Definition of the human raf amino-terminal regulatory region by deletion mutagenesis. $\mathrm{Mol}$ Cell Biol. (1989) 9:639-47. doi: 10.1128/MCB.9.2.639

71. Cutler RE Jr, Stephens RM, Saracino MR, Morrison DK. Autoregulation of the Raf-1 serine/threonine kinase. Proc Natl Acad Sci USA. (1998) 95:92149. doi: 10.1073/pnas.95.16.9214

72. Tran NH, Frost JA. Phosphorylation of Raf-1 by p21-activated kinase 1 and Src regulates Raf-1 autoinhibition. J Biol Chem. (2003) 278:112216. doi: 10.1074/jbc.M210318200

73. Tran NH, Wu X, Frost JA. B-Raf and Raf-1 are regulated by distinct autoregulatory mechanisms. J Biol Chem. (2005) 280:16244-53. doi: 10.1074/jbc.M501185200

74. Zhang BH, Guan KL. Activation of B-Raf kinase requires phosphorylation of the conserved residues Thr598 and Ser601. EMBO J. (2000) 19:542939. doi: 10.1093/emboj/19.20.5429

75. Zhang BH, Guan KL. Regulation of the Raf kinase by phosphorylation. Exp Lung Res. (2001) 27:269-95. doi: 10.1080/0190214013 00054046

76. Wan PT, Garnett MJ, Roe SM, Lee S, Niculescu-Duvaz D, Good VM, et al. Mechanism of activation of the RAF-ERK signaling pathway by oncogenic mutations of B-RAF. Cell. (2004) 116:855-67. doi: 10.1016/S0092-8674(04)00215-6

77. Kohler M, Roring M, Schorch B, Heilmann K, Stickel N, Fiala GJ, et al. Activation loop phosphorylation regulates B-Raf in vivo and transformation by B-Raf mutants. EMBO J. (2016) 35:143-61. doi: 10.15252/embj.2015 92097

78. Mason CS, Springer CJ, Cooper RG, Superti-Furga G, Marshall CJ, Marais R. Serine and tyrosine phosphorylations cooperate in Raf-1, but not B-Raf activation. EMBO J. (1999) 18:2137-48. doi: 10.1093/emboj/18.8.2137

79. Cook SJ, Mccormick F. Inhibition by cAMP of Ras-dependent activation of Raf. Science. (1993) 262:1069-72. doi: 10.1126/science.7694367

80. Dhillon AS, Meikle S, Yazici Z, Eulitz M, Kolch W. Regulation of Raf1 activation and signalling by dephosphorylation. EMBO J. (2002) 21:6471. doi: 10.1093/emboj/21.1.64

81. Wu J, Dent P, Jelinek T, Wolfman A, Weber MJ, Sturgill TW. Inhibition of the EGF-activated MAP kinase signaling pathway by adenosine 3',5'-monophosphate. Science. (1993) 262:1065-9. doi: 10.1126/science.76 94366

82. Rommel C, Clarke BA, Zimmermann S, Nunez L, Rossman R, Reid K, et al. Differentiation stage-specific inhibition of the Raf-MEK-ERK pathway by Akt. Science. (1999) 286:1738-41. doi: 10.1126/science.286.5445.1738

83. Zimmermann S, Moelling K. Phosphorylation and regulation of Raf by Akt (protein kinase B). Science. (1999) 286:17414. doi: 10.1126/science.286.5445.1741

84. Dhillon AS, Pollock C, Steen H, Shaw PE, Mischak H, Kolch W. Cyclic AMP-dependent kinase regulates Raf-1 kinase mainly by phosphorylation of serine 259. Mol Cell Biol. (2002) 22:3237-46. doi: 10.1128/MCB.22.10.3237-3246.2002

85. Light Y, Paterson H, Marais R. 14-3-3 antagonizes Ras-mediated Raf-1 recruitment to the plasma membrane to maintain signaling fidelity. Mol Cell Biol. (2002) 22:4984-96. doi: 10.1128/MCB.22.14.4984-4996.2002

86. Michaud NR, Fabian JR, Mathes KD, Morrison DK. 14-3-3 is not essential for Raf-1 function: identification of Raf-1 proteins that are biologically activated in a 14-3-3- and Ras-independent manner. Mol Cell Biol. (1995) 15:3390-7. doi: 10.1128/MCB.15.6.3390

87. Tzivion G, Luo Z, Avruch J. A dimeric 14-3-3 protein is an essential cofactor for Raf kinase activity. Nature. (1998) 394:88-92. doi: 10.1038/27938

88. Herrmann C, Horn G, Spaargaren M, Wittinghofer A. Differential interaction of the ras family GTP-binding proteins H-Ras, Rap1A, and R-Ras with the putative effector molecules Raf kinase and Ral-guanine nucleotide exchange factor. J Biol Chem. (1996) 271:6794-800. doi: 10.1074/jbc.271. 12.6794

89. Li S, Jang H, Zhang J, Nussinov R. Raf-1 Cysteine-rich domain increases the affinity of K-Ras/Raf at the membrane, promoting MAPK signaling. Structure. (2018) 26:513-25 e2. doi: 10.1016/j.str.2018.01.011

90. Li ZL, Prakash P, Buck M. A “Tug of War" maintains a dynamic proteinmembrane complex: molecular dynamics simulations of C-Raf RBD-CRD 
bound to K-Ras4B at an anionic membrane. ACS Cent Sci. (2018) 4:298305. doi: 10.1021/acscentsci.7b00593

91. Travers T, Lopez CA, Van QN, Neale C, Tonelli M, Stephen AG, Gnanakaran S. Molecular recognition of RAS/RAF complex at the membrane: role of RAF cysteine-rich domain. Sci Rep. (2018) 8:8461. doi: 10.1038/s41598-018-2 6832-4

92. Improta-Brears $\mathrm{T}$, Ghosh $\mathrm{S}$, Bell RM. Mutational analysis of Raf-1 cysteine rich domain: requirement for a cluster of basic aminoacids for interaction with phosphatidylserine. Mol Cell Biochem. (1999) 198:171-8. doi: 10.1023/A:100698 1411691

93. Thapar R, Williams JG, Campbell SL. NMR characterization of fulllength farnesylated and non-farnesylated $\mathrm{H}$-Ras and its implications for Raf activation. J Mol Biol. (2004) 343:1391-408. doi: 10.1016/j.jmb.2004. 08.106

94. Li ZL, Buck M. Computational modeling reveals that signaling lipids modulate the orientation of K-Ras $4 \mathrm{~A}$ at the membrane reflecting protein topology. Structure. (2017) 25:679-89 e2. doi: 10.1016/j.str.2017.02.007

95. Abankwa D, Gorfe AA, Inder K, Hancock JF. Ras membrane orientation and nanodomain localization generate isoform diversity. Proc Natl Acad Sci USA. (2010) 107:1130-5. doi: 10.1073/pnas.0903907107

96. Gorfe AA, Hanzal-Bayer M, Abankwa D, Hancock JF, Mccammon JA. Structure and dynamics of the full-length lipid-modified H-Ras protein in a 1,2-dimyristoylglycero-3-phosphocholine bilayer. J Med Chem. (2007) 50:674-84. doi: 10.1021/jm061053f

97. Muslin AJ, Tanner JW, Allen PM, Shaw AS. Interaction of 14-3-3 with signaling proteins is mediated by the recognition of phosphoserine. Cell. (1996) 84:889-97. doi: 10.1016/S0092-8674(00)81067-3

98. Rommel C, Radziwill G, Lovric J, Noeldeke J, Heinicke T, Jones D, et al. Activated Ras displaces 14-3-3 protein from the amino terminus of c-Raf-1. Oncogene. (1996) 12:609-19.

99. Abraham D, Podar K, Pacher M, Kubicek M, Welzel N, Hemmings BA, et al. Raf-1-associated protein phosphatase $2 \mathrm{~A}$ as a positive regulator of kinase activation. J Biol Chem. (2000) 275:22300-4. doi: 10.1074/jbc.M003259200

100. Jaumot M, Hancock JF. Protein phosphatases 1 and $2 \mathrm{~A}$ promote Raf-1 activation by regulating 14-3-3 interactions. Oncogene. (2001) 20:394958. doi: 10.1038/sj.onc. 1204526

101. Ory S, Zhou M, Conrads TP, Veenstra TD, Morrison DK. Protein phosphatase $2 \mathrm{~A}$ positively regulates Ras signaling by dephosphorylating KSR1 and Raf-1 on critical 14-3-3 binding sites. Curr Biol. (2003) 13:135664. doi: 10.1016/S0960-9822(03)00535-9

102. Matallanas D, Birtwistle M, Romano D, Zebisch A, Rauch J, Von Kriegsheim A, Kolch W. Raf family kinases: old dogs have learned new tricks. Genes Cancer. (2011) 2:232-60. doi: 10.1177/1947601911407323

103. Dumaz N, Marais R. Protein kinase A blocks Raf- 1 activity by stimulating 14-3-3 binding and blocking Raf-1 interaction with Ras. J Biol Chem. (2003) 278:29819-23. doi: 10.1074/jbc.C300182200

104. Molzan M, Ottmann C. Synergistic binding of the phosphorylated S233- and S259-binding sites of C-RAF to one 14-3-3zeta dimer. J Mol Biol. (2012) 423:486-95. doi: 10.1016/j.jmb.2012.08.009

105. MEK Binding to KSR promotes allosteric activation of BRAF. Cancer Discov. (2018) 8:385. doi: 10.1158/2159-8290.CD-RW2018-033

106. Lavoie H, Sahmi M, Maisonneuve P, Marullo SA, Thevakumaran N, Jin T, et al. MEK drives BRAF activation through allosteric control of KSR proteins. Nature. (2018) 554:549-53. doi: 10.1038/nature25478

107. Ren JG, Li Z, Sacks DB. IQGAP1 modulates activation of B-Raf. Proc Natl Acad Sci USA. (2007) 104:10465-9. doi: 10.1073/pnas.06113 08104

108. Stewart S, Sundaram M, Zhang Y, Lee J, Han M, Guan KL. Kinase suppressor of Ras forms a multiprotein signaling complex and modulates MEK localization. Mol Cell Biol. (1999) 19:5523-34. doi: 10.1128/MCB.19.8.5523

109. Shalom-Feuerstein R, Plowman SJ, Rotblat B, Ariotti N, Tian $\mathrm{T}$, Hancock JF, et al. K-ras nanoclustering is subverted by overexpression of the scaffold protein galectin-3. Cancer Res. (2008) 68:6608-16. doi: 10.1158/0008-5472.CAN-08-1117

110. White CD, Erdemir HH, Sacks DB. IQGAP1 and its binding proteins control diverse biological functions. Cell Signal. (2012) 24:826-34. doi: 10.1016/j.cellsig.2011.12.005
111. Nussinov R, Tsai CJ, Jang H. Is Nanoclustering essential for all oncogenic KRas pathways? Can it explain why wild-type KRas can inhibit its oncogenic variant? Semin Cancer Biol. (2019) 54:114-20. doi: 10.1016/j.semcancer.2018.01.002

112. Santos E, Crespo P. The RAS-ERK pathway: a route for couples. Sci Signal. (2018) 11:eaav0917. doi: 10.1126/scisignal.aav0917

113. Nussinov R, Jang H. Dynamic multiprotein assemblies shape the spatial structure of cell signaling. Prog Biophys Mol Biol. (2014) 116:15864. doi: 10.1016/j.pbiomolbio.2014.07.002

114. Avruch J, Xavier R, Bardeesy N, Zhang XF, Praskova M, Zhou D, et al. Rassf family of tumor suppressor polypeptides. J Biol Chem. (2009) 284:11001-5. doi: 10.1074/jbc.R80 0073200

115. Avruch J, Zhou D, Fitamant J, Bardeesy N, Mou F, Barrufet LR. Protein kinases of the Hippo pathway: regulation and substrates. Semin Cell Dev Biol. (2012) 23:770-84. doi: 10.1016/j.semcdb.2012.07.002

116. Donninger H, Schmidt ML, Mezzanotte J, Barnoud T, Clark GJ. Ras signaling through RASSF proteins. Semin Cell Dev Biol. (2016) 58:8695. doi: 10.1016/j.semcdb.2016.06.007

117. Nussinov R, Jang H, Tsai CJ, Liao TJ, Li S, Fushman D, et al. Intrinsic protein disorder in oncogenic KRAS signaling. Cell Mol Life Sci. (2017) 74:3245-61. doi: 10.1007/s00018-017-2564-3

118. Richter AM, Pfeifer GP, Dammann RH. The RASSF proteins in cancer; from epigenetic silencing to functional characterization. Biochim Biophys Acta. (2009) 1796:114-28. doi: 10.1016/j.bbcan.2009.03.004

119. Fallahi E, O'driscoll NA, Matallanas D. The MST/hippo pathway and cell death: a non-canonical affair. Genes. (2016) 7:E28. doi: 10.3390/genes7 060028

120. Huang CH, Mandelker D, Schmidt-Kittler O, Samuels Y, Velculescu VE, Kinzler KW, et al. The structure of a human p110alpha/p85alpha complex elucidates the effects of oncogenic PI3Kalpha mutations. Science. (2007) 318:1744-8. doi: 10.1126/science.1150799

121. Makbul C, Constantinescu Aruxandei D, Hofmann E, Schwarz D, Wolf E, Herrmann C. Structural and thermodynamic characterization of Nore1SARAH: a small, helical module important in signal transduction networks. Biochemistry. (2013) 52:1045-54. doi: 10.1021/bi3014642

122. Stieglitz B, Bee C, Schwarz D, Yildiz O, Moshnikova A, Khokhlatchev A, et al. Novel type of Ras effector interaction established between tumour suppressor NORE1A and Ras switch II. EMBO J. (2008) 27:1995-2005. doi: 10.1038/emboj.20 08.125

123. Kumar S, Ma B, Tsai CJ, Sinha N, Nussinov R. Folding and binding cascades: dynamic landscapes and population shifts. Protein Sci. (2000) 9:10-9. doi: 10.1110/ps.9.1.10

124. Liu J, Nussinov R. Energetic redistribution in allostery to execute protein function. Proc Natl Acad Sci USA. (2017) 114:7480-2. doi: 10.1073/pnas.1709071114

125. Ma B, Kumar S, Tsai CJ, Nussinov R. Folding funnels and binding mechanisms. Protein Eng. (1999) 12:713-20. doi: 10.1093/protein/12.9.713

126. Nussinov R, Tsai CJ, Csermely P. Allo-network drugs: harnessing allostery in cellular networks. Trends Pharmacol Sci. (2011) 32:686-93. doi: 10.1016/j.tips.2011. 08.004

127. Nussinov R, Wolynes PG. A second molecular biology revolution? The energy landscapes of biomolecular function. Phys Chem Chem Phys. (2014) 16:6321-2. doi: 10.1039/c4cp $90027 \mathrm{~h}$

128. Tsai CJ, Del Sol A, Nussinov R. Protein allostery, signal transmission and dynamics: a classification scheme of allosteric mechanisms. Mol Biosyst. (2009) 5:207-16. doi: 10.1039/b81 $9720 \mathrm{~b}$

129. Tsai CJ, Kumar S, Ma B, Nussinov R. Folding funnels, binding funnels, and protein function. Protein Sci. (1999) 8:1181-90. doi: 10.1110/ps.8.6.1181

130. Tsai CJ, Ma B, Nussinov R. Folding and binding cascades: shifts in energy landscapes. Proc Natl Acad Sci USA. (1999) 96:9970-2. doi: 10.1073/pnas.96.18.9970

131. Wei G, Xi W, Nussinov R, Ma B. Protein ensembles: how does nature harness thermodynamic fluctuations for life? the 
diverse functional roles of conformational ensembles in the cell. Chem Rev. (2016) 116:6516-51. doi: 10.1021/acs.chemrev.5b 00562

132. Tsai CJ, Del Sol A, Nussinov R. Allostery: absence of a change in shape does not imply that allostery is not at play. J Mol Biol. (2008) 378:111. doi: $10.1016 /$ j.jmb.2008.02.034

133. Yang $\mathrm{H}$, Jiang $\mathrm{X}$, Li $\mathrm{B}$, Yang $\mathrm{HJ}$, Miller $\mathrm{M}$, Yang $\mathrm{A}$, Dhar $\mathrm{A}$, Pavletich NP. Mechanisms of mTORC1 activation by RHEB and inhibition by PRAS40. Nature. (2017) 552:368-73. doi: 10.1038/nature 25023
Conflict of Interest: The authors declare that the research was conducted in the absence of any commercial or financial relationships that could be construed as a potential conflict of interest.

Copyright $(02019$ Nussinov, Tsai and Jang. This is an open-access article distributed under the terms of the Creative Commons Attribution License (CC BY). The use, distribution or reproduction in other forums is permitted, provided the original author(s) and the copyright owner(s) are credited and that the original publication in this journal is cited, in accordance with accepted academic practice. No use, distribution or reproduction is permitted which does not comply with these terms. 